\title{
Zhike pingchuan granules improve bronchial asthma by regulating the IL-6/JAK2/STAT3 pathway
}

\author{
YUMEI REN ${ }^{1 *}$, YULING LIU $^{2 *}$, SHOUCHUAN WANG ${ }^{3,4}$, ZHEN LEI $^{5}$, YONGBIN YAN $^{6}$, \\ XUTAO GUAN $^{7}$, JIANGHONG HOU ${ }^{8}$, SHAN ZHU ${ }^{9}$, HAIJUN SHAN $^{9}$, XINLEI TIAN $^{9}$, \\ QUAN WANG $^{10}$, CAIHONG CAO $^{9}$, YINGYING ZHANG ${ }^{9}$ and YUNFENG MA ${ }^{11}$
}

\begin{abstract}
${ }^{1}$ Pediatric Department of The Second Clinical Medical College of Henan University of Traditional Chinese Medicine, Zhengzhou, Henan 450000; ${ }^{2}$ Pediatric Department of Nanjing Pukou Hospital of Chinese Medicine, Nanjing, Jiangsu 211800; ${ }^{3}$ Pediatric Department of Jiangsu Province Hospital of Chinese Medicine, Nanjing, Jiangsu 210029;

${ }^{4}$ The First Clinical Medical College of Nanjing University of Chinese Medicine, Nanjing, Jiangsu 210023;

${ }^{5}$ Central Laboratory of The Second Affiliated Hospital of Henan University of Traditional Chinese Medicine, Zhengzhou, Henan 450002; ${ }^{6}$ Pediatric Department of The First Affiliated Hospital of Henan University of Traditional Chinese Medicine;

${ }^{7}$ Oncology Department of The First Affiliated Hospital of Henan University of Traditional Chinese Medicine, Zhengzhou,

Henan 450000; ${ }^{8}$ Health Preserving Discipline of The Second Affiliated Hospital of Henan University of Traditional Chinese Medicine; ${ }^{9}$ Pediatric Department of The Second Affiliated Hospital of Henan University of Traditional Chinese Medicine, Zhengzhou, Henan 450002; ${ }^{10}$ Experimental Center of Henan University of Chinese Medicine, Zhengzhou, Henan 450046; ${ }^{11}$ Discipline of Bone Injury of The Second Clinical Medical College of Henan University of Traditional Chinese Medicine, Zhengzhou, Henan 450000, P.R. China
\end{abstract}

Received June 16, 2020; Accepted May 25, 2021

DOI: $10.3892 / \mathrm{etm} .2021 .10331$

\begin{abstract}
The present study aimed to investigate the effects of zhike pingchuan granules (ZKPC) on bronchial asthma and the underlying mechanism. A bronchial asthma mouse model was established by aerosol inhalation of ovalbumin. The changes in lung pathomorphology were observed by hematoxylin and eosin staining. The levels of IL-1 $\beta$, TNF- $\alpha$ and IL-6 in bronchoalveolar lavage fluid (BALF) and serum were detected by corresponding ELISA kits. Levels of reactive oxygen species, malondialdehyde and superoxide dismutase in lung tissues were analyzed using corresponding kits. The expression of proteins related to apoptosis and the IL-6/janus kinase 2 (JAK2)/STAT3 pathway was detected by western blot analysis. The results showed that ZKPC significantly restored the dry/wet ratio and alleviated lung pathomorphology of bronchial asthmatic mice. In addition, ZKPC inhibits inflammation, oxidative stress levels and cell
\end{abstract}

Correspondence to: Mr. Yunfeng Ma, Discipline of Bone Injury of The Second Clinical Medical College of Henan University of Traditional Chinese Medicine, 6 Dongfeng Road, Zhengzhou, Henan 450000, P.R. China

E-mail: myunfeng123@163.com

${ }^{*}$ Contributed equally

Key words: zhike pingchuan granules, bronchial asthma, IL-6/janus kinase 2/STAT3 pathway, inflammation apoptosis in bronchial asthmatic mice and also suppressed the IL-6/JAK2/STAT3 pathway. Fedratinib (a JAK2 inhibitor) further strengthened the alleviative effects of ZKPC on bronchial asthma. In conclusion, ZKPC improved bronchial asthma by suppressing the IL-6/JAK2/STAT3 pathway.

\section{Introduction}

Bronchial asthma is a heterogeneous disease characterized by chronic airway inflammation and airway responsiveness (1). The disease can occur at any age, but is more common in children and is a common cause of pediatric emergency worldwide (2). Approximately one-third of children present with wheezing before the age of two, and about one-fifths of children present with recurrent or persistent wheezing (3). The number of children with bronchial asthma in China is reported to be about 30 million (4). Childhood asthma in the young age group (aged $<5$ years) in developing countries is often misdiagnosed as pneumonia without effective treatment, leading to increased morbidity and mortality in this age group (5). Although the rate of bronchial asthma in China has risen from $28.7 \%$ in 2008 to $39.2 \%$ in 2016, it remains at a low level (6). With the development of traditional Chinese medicine (TCM) research, the advantages of TCM in the treatment of childhood bronchial asthma have been clinically valued (7). Therefore, it is of importance to find effective methods or drugs to treat childhood bronchial asthma from the perspective of TCM.

TCM has been widely used and demonstrated to be effective in the treatment of bronchial asthma (8-10). Zhike pingchuan granules (ZKPC) play a role in relieving coughing and reducing 
sputum to relieve asthma. Their ingredients include Gingko, Lumbricus, Perilla leaves, rhizome Pinelliae preparata, Mibaibu stemona root, Radix asteris, Folium eriobotryae, Platycodon grandiflorus, Scutellaria baicalensis, apricot kernel, peach kernel, Cortex mori and Digupi. Jin et al (11) demonstrated that Ginkgo lactone showed anti-inflammatory and anti-oxidant effects in a rat model of $\mathrm{A} \beta_{1-40}$ induced Alzheimer's disease, improving nerve injury and cognitive function. Thorpe et al (12) indicated that the Gingko biloba extract EGb 761 could inhibit inflammation and thermal hyperalgesia for the treatment of inflammatory pain. Wuwei dilong decoction could inhibit the infiltration and spread of inflammatory cells in asthma (13). In addition, the active ingredients of Aster tataricus and Pinellia ternata were both demonstrated to have anti-inflammatory properties $(14,15)$.

Airway inflammation is an important factor in the development of bronchial asthma. IL-6 is synthesized and secreted by lymphocytes and mononuclear macrophages after activation and is related to local inflammatory response, participates in the body's inflammatory damage process and is also the main inflammatory factor of bronchial asthma $(16,17)$. Janus kinase 2 (JAK2)/STAT3 signaling plays an important role in various biological activities, such as tumorigenesis and inflammation (18). JAK2 can phosphorylate STAT3, which activates STAT3 and downstream target genes (19). Studies have shown that JAK2/STAT3 signaling is crucial for the pathogenesis of asthma $(20,21)$. Furthermore, Simon et al (22) found that STAT3 inhibition could suppress proliferation of airway smooth muscle cells in asthma. Therefore, the JAK2/STAT3 signaling pathway plays crucial roles in the development and progression of asthma. Yan et al (23) demonstrated that JAK2 is associated with airway remodeling in bronchial asthma. Shi et al (24) indicated that IL-6 could promote the activation of the JAK2/STAT3 pathway.

Therefore, the present study aimed to investigate the therapeutic effects of ZKPC on bronchial asthma, as well as the underlying mechanism related to the IL-6/JAK2/STAT3 pathway.

\section{Materials and methods}

Mouse model of bronchial asthma. A total of 60 male C57BL/6J mice (specific-pathogen-free; age, 6-8 weeks; weight range, 16-20 g) were purchased from Beijing Vital River Laboratory Animal Technology Co., Ltd., and were maintained under a 12-h light/dark cycle at room temperature $\left(22 \pm 2^{\circ} \mathrm{C}\right)$ with a humidity of $45 \%$ and allowed ad libitum access to water and food. Each group contained 10 mice. Normal saline $(5 \mathrm{ml})$ was suspended with $200 \mathrm{mg}$ aluminum hydroxide to prepare a $4 \%$ aluminum hydroxide gel. A total of $1.5 \mathrm{mg}$ ovalbumin (OVA; Thermo Scientific Fisher, Inc.) was fully dissolved in $2.5 \mathrm{ml}$ normal saline, which was then suspended in $2.5 \mathrm{ml} 4 \%$ aluminum hydroxide gel to prepare the sensitizing solution. Mice was intraperitoneally injected with $0.2 \mathrm{ml} /$ day of newly prepared sensitizing solution for 15 days in total. ZKPC granules (manufactured by Henan Shizhen Pharmaceutical Co., Ltd.) were purchased from the Second Affiliated Hospital of Henan University of Chinese Medicine. ZKPC solution was prepared by mixing ZKPC granules with distilled water to obtain a final ZKPC solution concentration of $0.1 \mathrm{~g} / \mathrm{ml}$.
In the first set of experiments, from days 15-30, the mice in the Model group were placed in a transparent airtight chamber and inhaled aerosolized $1 \%$ OVA solution naturally for $30 \mathrm{~min} /$ day $(\mathrm{n}=10)$. Mice in the control group were treated with normal saline in the same manner $(\mathrm{n}=10)$.

In the ZKPC-Low (L) group, mice were administered with equivalent doses of ZKPC $(0.525 \mathrm{~g} / \mathrm{kg})$ by gavage $30 \mathrm{~min}$ prior to aerosol inhalation $(\mathrm{n}=10)$. In the ZKPC-High $(\mathrm{H})$ group, mice were administered with ZKPC at four times the equivalent dosage $(2.1 \mathrm{~g} / \mathrm{kg})$ by gavage $30 \mathrm{~min}$ prior to aerosol inhalation $(\mathrm{n}=10)$. At $24 \mathrm{~h}$ after the last aerosol inhalation, mice were anesthetized via intraperitoneal injection of $1 \%$ pentobarbital sodium $(40 \mathrm{mg} / \mathrm{kg})$, and blood from the eyeballs $(0.2-0.3 \mathrm{ml})$ and bronchoalveolar lavage fluid (BALF) were obtained. Subsequently, anaesthetized mice were killed by cervical dislocation and lung tissues were extracted from mice after confirmation of cardiac arrest. The control group and model group were given equal amounts of distilled water.

In the second set of experiments, mice in the fedratinib (Fedr) group were administered with Fedr $(60 \mathrm{mg} / \mathrm{kg}$ ) by gavage 30 min prior to aerosol inhalation $(\mathrm{n}=10)$. In the ZKPC + Fedr group, mice were administered with Fedr $(60 \mathrm{mg} / \mathrm{kg})$ and ZKPC at four times the equivalent dosage $(2.1 \mathrm{~g} / \mathrm{kg})$ by gavage 30 min prior to aerosol inhalation $(n=10)$. The dose of Fedr was determined and modified based on a previous study (25). Mice health and behavior were monitored every day. All animal experiments were approved by the Animal Experimental Ethics Committee of Henan University of Traditional Chinese Medicine (approval no. 20190412WZ).

Dry/wet $(D / W)$ ratio of the lungs. The lower lobe of the right lung was taken, of which the wet weight was recorded. The lung dry weight was recorded after baking in an oven for $72 \mathrm{~h}$. The $\mathrm{D} / \mathrm{W}$ ratio of lung was then calculated.

Hematoxylin and eosin $(H \& E)$ staining. Lung tissue was collected, fixed with $10 \%$ formalin solution at $4^{\circ} \mathrm{C}$ for $>24 \mathrm{~h}$ and dehydrated in different concentrations (low to high) of ethanol. Samples were then embedded in paraffin, which were cut into $5 \mu \mathrm{m}$ sections. Following hematoxylin staining for $3 \mathrm{~min}$ and eosin staining for $3 \mathrm{~min}$ at room temperature, the slices were sealed. Finally, the pathological morphology of mouse lung tissue was observed under a full-field pathological slice scanner.

ELISA. BALF and serum (stored at $-80^{\circ} \mathrm{C}$ ) were thawed. The levels of IL-1 $\beta$, TNF- $\alpha$ and IL- 6 in BALF and serum were detected by IL- $1 \beta$ ELISA kit (cat. no. PI301), TNF- $\alpha$ ELISA kit (cat. no. PT512) and IL-6 ELISA kit (cat. no. PI326), respectively, according to the manufacturer's protocol (Beyotime Institute of Biotechnology).

Detection of oxidative stress factors. Part of the lung tissue was prepared into $10 \%$ tissue homogenate, which was centrifuged at $10,000 \mathrm{x}$ g for $20 \mathrm{~min}$ at $4^{\circ} \mathrm{C}$. The supernatant was collected and tested with reactive oxygen species (ROS; cat. no. E-BC-K138-F; Elabscience Biotechnology Inc.), malondialdehyde (MDA; cat. no. S0131S; Beyotime Institute of Biotechnology) and superoxide dismutase (SOD; cat. no. S0109; Beyotime Institute of Biotechnology) kits. 


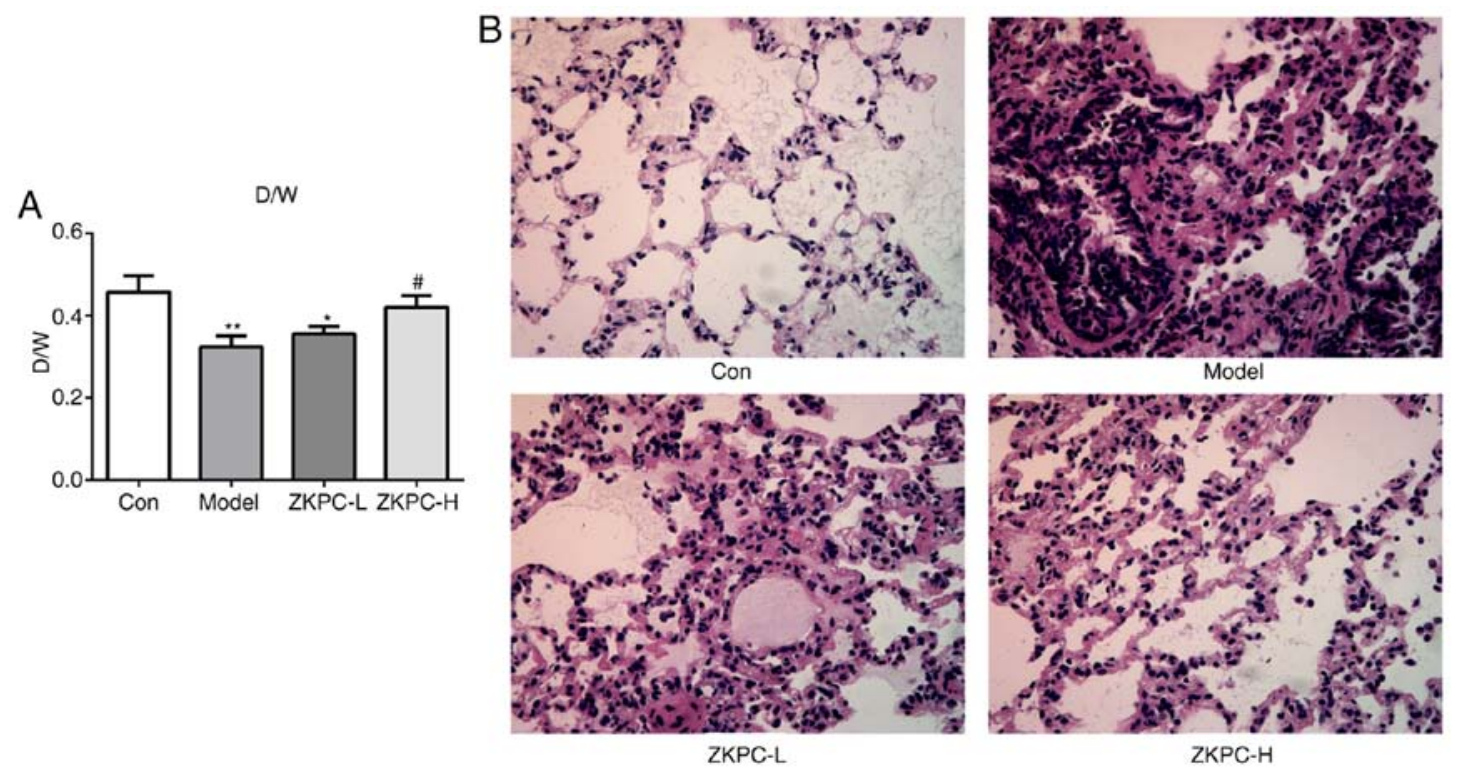

Figure 1. ZKPC improves bronchial asthma-induced lung injury. (A) D/W ratio of lung tissues was calculated after weighing. "P<0.05 and ** $\mathrm{P}<0.01$ vs. Con group. " $\mathrm{P}<0.05$ vs. Model group. (B) Lung pathomorphology was observed by hematoxylin and eosin staining (magnification, $\mathrm{x} 400$ ). $\mathrm{n}=10$. $\mathrm{D} / \mathrm{W}$, dry/weigh; Con, control; ZKPC, zhike pingchuan granules; L, low; H, high.

Western blot analysis. Lung tissue samples were homogenized using RIPA buffer and centrifuged at $10,000 \mathrm{x} \mathrm{g}$ for $10 \mathrm{~min}$ at $4^{\circ} \mathrm{C}$. Protein concentration was determined using the BCA method. Total protein $(25 \mu \mathrm{g})$ were loaded per lane and separated via 10\% SDS-PAGE, followed transfer to PVDF membranes. Subsequently, membranes were blocked in $5 \%$ non-fat milk in TBS-Tween-20 (0.05\%) buffer for $1 \mathrm{~h}$ at $25^{\circ} \mathrm{C}$ and incubated with primary antibodies overnight at $4^{\circ} \mathrm{C}$. Membranes were then incubated with rabbit IgG horseradish peroxidase-conjugated secondary antibody (cat. no. 7074; dilution, 1:1,000; Cell Signaling Technologies, Inc.) for $1 \mathrm{~h}$ at $25^{\circ} \mathrm{C}$. The primary antibodies (all from Cell Signaling Technology, Inc.) used were as follows: Anti-IL-6 (cat. no. 12912; dilution, 1:1,000), anti-Bcl2 (cat. no. 3498; dilution, 1:1,000), anti-Bax (cat. no. 2772; dilution, 1:1,000), anti-cleaved (c) caspase-3 (cat. no. 9661; dilution, 1:1,000), anti-caspase-3 (cat. no. 9662; dilution, 1:1,000), anti-JAK2 (cat. no. 3230; dilution, 1:1,000), anti-phosphorylated (p)-JAK2 (cat. no. 3771; dilution, 1:1,000), anti-STAT3 (cat. no. 12640; dilution, 1:1,000), anti-p-STAT3 (cat. no. 9145; dilution, 1:2,000) and anti-GAPDH (cat. no. 5174; dilution, 1:1,000). Bands were visualized using an enhanced chemiluminescence system (Amersham Biosciences; Cytiva) and band gray values were semi-quantified using Image J software (version 1.0; National Institutes of Health).

Statistical analysis. Data were represented by the mean \pm SD SPSS 20.0 (IBM Corp.) was used to conduct one-way ANOVA and Tukey's post hoc test. $\mathrm{P}<0.05$ was considered to indicate a statistically significant difference. All experiments were replicated three times.

\section{Results}

ZKPC improves lung injury induced by bronchial asthma. The D/W ratio of model group was significantly lower compared with the control group. In the ZKPC-H group, the $\mathrm{D} / \mathrm{W}$ ratio was significantly increased compared with the model group (Fig. 1A). In the model group, alveolar spaces presented with obvious hyperemia, bleeding and cell infiltration, and alveolar walls were not clearly observed. Upon increasing concentrations of ZKPC, hyperemia, bleeding and cell infiltration in tissue interspace and alveolar cavity were markedly decreased, and the structure of most alveolar walls were complete in the ZKPC-H group (Fig. 1B).

ZKPC improves inflammation and oxidative stress in bronchial asthma mice. As shown in Fig. $2 \mathrm{~A}$, the levels of IL-1 $\beta$, TNF- $\alpha$ and IL-6 were significantly upregulated in BALF, which was gradually suppressed by increased concentrations of ZKPC. The levels of IL-1 $\beta$, TNF- $\alpha$ and IL- 6 in serum showed similar changes to that in BALF (Fig. 2B). As shown in Fig. 2C-E, the levels of ROS and MDA increased while SOD levels decreased in lung tissues of the model group, and ZKPC effectively reversed the levels of ROS, MDA and SOD.

ZKPC inhibits cell apoptosis and the IL-6/JAK2/STAT3 pathway in lung tissue. The expression levels of IL-6, Bax and c-caspase-3 increased while Bcl-2 expression levels declined in lung tissues of model mice. Upon increasing concentrations of ZKPC, the expression levels of IL-6, Bax and c-caspase-3 were gradually downregulated while $\mathrm{Bcl}-2$ expression levels were upregulated in lung tissues (Fig. 3A). p-JAK2 and p-STAT3 levels in lung tissues of model mice was upregulated, which was partially reversed by increasing concentrations of ZKPC (Fig. 3B).

Fedratinib enhances the improvement effects of ZKPC on bronchial asthma-induced lung injury. Fedratinib treatment upregulated the $\mathrm{D} / \mathrm{W}$ ratio compared with the model group, and the $\mathrm{D} / \mathrm{W}$ ratio was further increased with co-treatment of ZKPC and fedratinib (Fig. 4A). Lung injury in Fedr group was alleviated while the degree of improvement was lower 

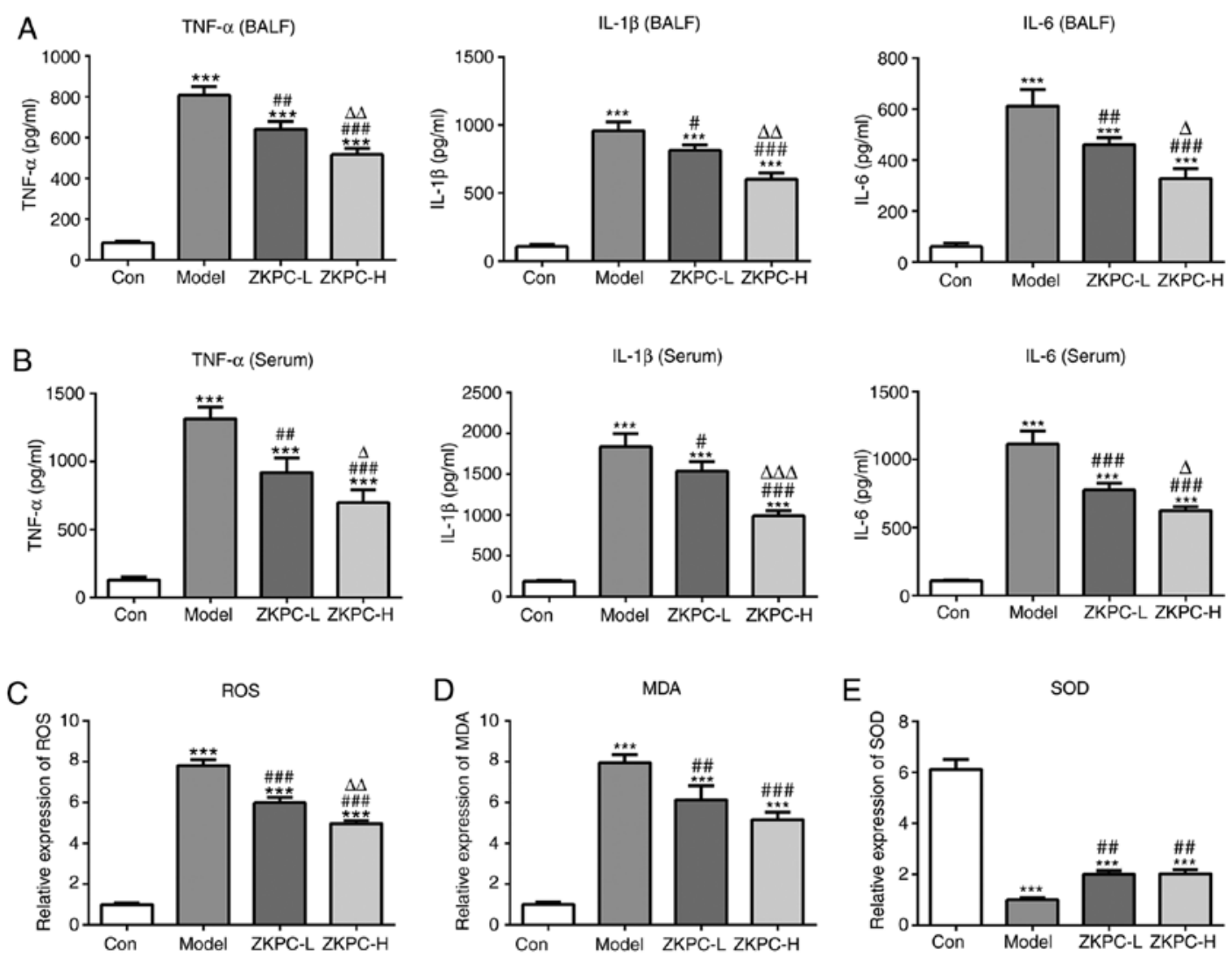

Figure 2. ZKPC improves inflammation and oxidative stress in bronchial asthmatic mice. The levels of IL-1 $\beta$, TNF- $\alpha$ and IL-6 in (A) BALF and (B) serum were analyzed by ELISA. (C) ROS levels in lung tissues was detected using a ROS kit. (D) MDA levels in lung tissues was detected using an MDA kit. (E) SOD levels in lung tissues was detected using a SOD kit. ${ }^{* * *} \mathrm{P}<0.001$ vs. Con group. ${ }^{\#} \mathrm{P}<0.05,{ }^{\# \#} \mathrm{P}<0.01$ and ${ }^{\# \# \#} \mathrm{P}<0.001$ vs. Model group. ${ }^{\Delta} \mathrm{P}<0.05$ and ${ }^{\Delta \Delta} \mathrm{P}<0.01$ and ${ }^{\Delta \triangle} \mathrm{P}<0.001$ vs. ZKPC-L group. $\mathrm{n}=10$. BALF, bronchoalveolar lavage fluid; ROS, reactive oxygen species; MDA, malondialdehyde; SOD, superoxide dismutase; ZKPC, zhike pingchuan granules; L, low; H, high; Con, control.

compared with the ZKPC group. Co-treatment of ZKPC and fedratinib showed the highest improvement effects on lung tissues (Fig. 4B).

Fedratinib enhances the improvement effects of ZKPC on inflammation and oxidative stress in bronchial asthmatic mice. Fedratinib treatment downregulated the levels of IL-1 $\beta$, TNF- $\alpha$ and IL-6, and co-treatment of ZKPC and fedratinib showed further inhibition effects on the levels of IL-1 $\beta$, TNF- $\alpha$ and IL- 6 in both BALF and serum (Fig. 5A and B). The levels of ROS and MDA decreased and SOD levels increased in lung tissues treated with fedratinib, and co-treatment of ZKPC and fedratinib further decreased levels of ROS and MDA, while SOD levels did not significantly change (Fig. 5C-E).

Fedratinib enhances the inhibitory effects of ZKPC on cell apoptosis and the IL-6/JAK2/STAT3 pathway in lung tissue. The expression levels of IL-6, Bax and c-caspase-3 declined, while Bcl-2 expression levels increased in lung tissues of model mice treated with fedratinib. Meanwhile, the expression on IL-6, Bax, c-caspase-3 and Bcl-2 were further declined by co-treatment of ZKPC and fedratinib (Fig. 6A). Fedratinib treatment downregulated p-JAK2 levels, which was further suppressed by co-treatment of ZKPC and fedratinib. p-STAT3 levels slightly decreased in the Fedr group and further decreased in ZKPC + Fedr group (Fig. 6B).

\section{Discussion}

Asthma is presented with repeated and reversible airway obstruction, which is often associated with airway hyperresponsiveness and inflammation (26). Although glucocorticoid drugs are the preferred treatment for alleviating asthma, there are many adverse effects caused glucocorticoids, which makes a safe dose difficult to determine. In addition, asthmatic patients are unable to inhale glucocorticoids daily, limiting the curative effect of glucocorticoids in asthma therapy $(27,28)$. Therefore, it is urgent to find newly identified drugs for the treatment of asthma that are highly efficacious, easily managed and with fewer adverse effects.

As a pre-inflammatory factor with multiple biological functions, IL-6 plays an important role in the proliferation and differentiation of immune-modulating inflammatory response cells, and is closely related to the incidence of inflammatory diseases, such as asthma and rheumatoid arthritis (29). IL-6 expression was shown to be high in the serum, lung tissues and BALF of asthmatic patients $(30,31)$. Kuhn et al (32) found that in mouse experiments, significant expression of IL-6 resulted in the expansion of alveolar cavities, followed by the infiltration of peripheral tracheal monocytes, which gradually resulted in the thickening of the airway wall, airway epithelial fibrosis and airway remodeling. The present study found increased levels of IL-6, IL- $1 \beta$ and TNF- $\alpha$ in the serum and BALF of bronchial asthmatic mice. In addition, ZKPC 

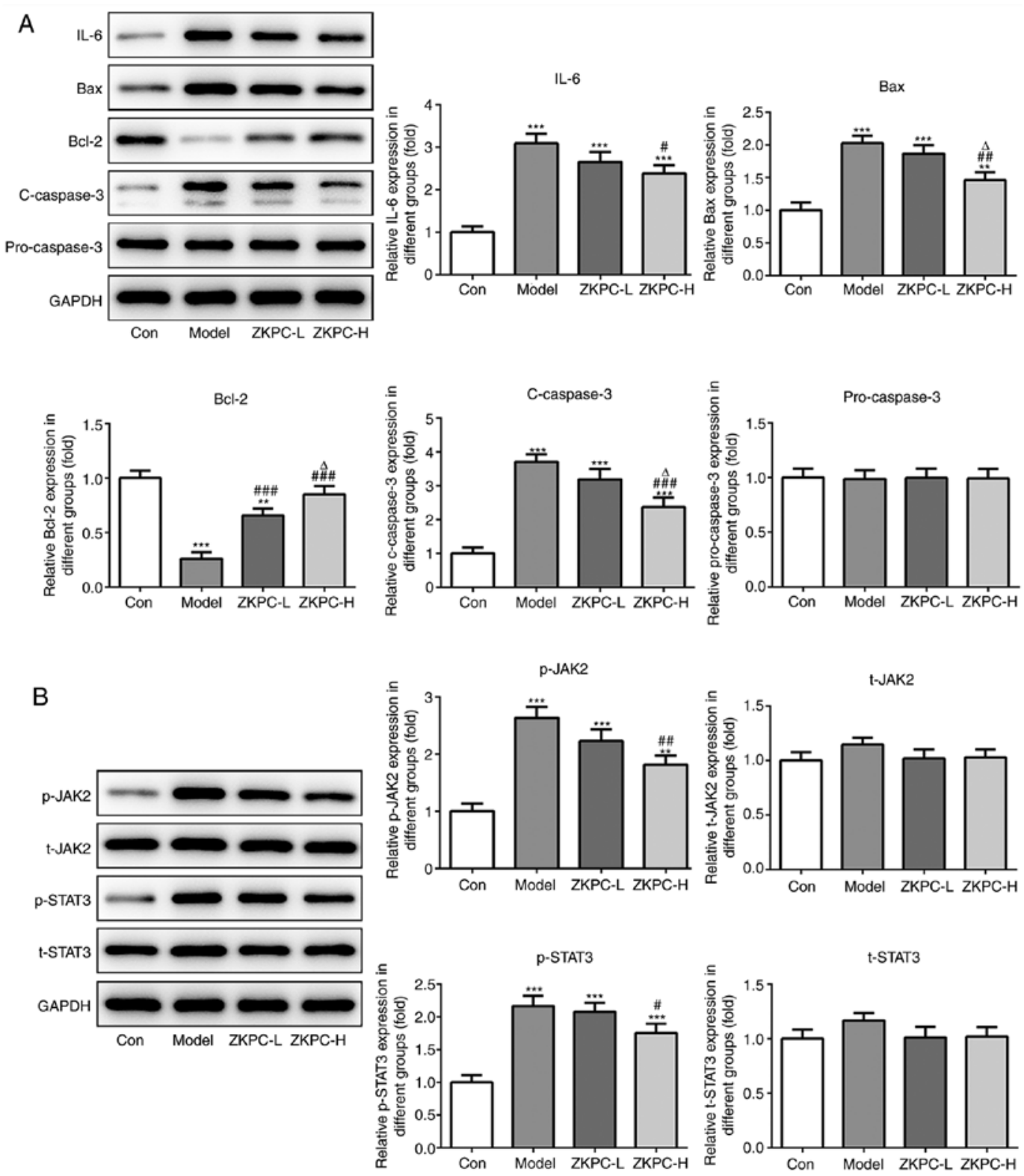

Figure 3.ZKPC inhibits cell apoptosis and the IL-6/JAK2/STAT3 pathway in lung tissue. (A) The expression of IL-6, Bax, Bcl-2, c-caspase-3 and pro-caspase-3 in lung and (B) levels of t-JAK2, p-JAK2, t-STAT3 and p-STAT3 in lung tissues were determined by Western blot analysis. ${ }^{* * *} \mathrm{P}<0.01$ and ${ }^{* * * *} \mathrm{P}<0.001$ vs. Con group. ${ }^{\#} \mathrm{P}<0.05,{ }^{\# \#} \mathrm{P}<0.01$ and ${ }^{\# \# \#} \mathrm{P}<0.001$ vs. Model group. ${ }^{\wedge} \mathrm{P}<0.05$ vs. ZKPC-L group. $\mathrm{n}=10$. $\mathrm{c}$, cleaved; $\mathrm{t}$, total; $\mathrm{p}$, phosphorylated; ZKPC, zhike pingchuan granules; L, low; H, high; Con, control.

treatment decreased the levels of IL-6, IL-1 $\beta$ and TNF- $\alpha$ in serum and BALF.

A study showed that STAT3 mediated the occurrence and development of wheezing and could reduce airway inflammation and remodeling by inhibiting the activation of STAT3 signaling (20). Gavino et al (33) found that STAT3 activation could lead to the accumulation of local inflammatory factors in the airway, hence aggravating the original airway inflammation. This indicated that inhibiting STAT3 expression could block the transmission of relevant inflammatory signals, effectively treat airway inflammation, control repeated wheezing and result in the prevention of asthma. Endogenous STAT3 could promote the ability of smooth muscle cells to generate blood vessels by activating endothelial growth factors in asthmatic patients, which could be a possible target for asthma treatment (34). JAK2 is demonstrated to be important in the pathogenesis of asthma (35). Gong et al (36) indicated that kaempferol suppressed eosinophil infiltration and airway inflammation in mice with allergic asthma by inhibiting JAK2. Nakano et al (37) found that niflumic acid alleviated IL-13-induced asthma in mice by suppressing JAK2. IL-6 can combine with soluble IL-6R to activate glycoprotein 130 of the cell membrane surface, thus activating JAK2/STAT3 signaling pathway (38). The present study confirmed that the expression levels of IL-6, p-JAK2 and p-STAT3 in lung tissues of bronchial asthmatic mice were significantly increased, which were downregulated by ZKPC treatment. Fedratinib, a JAK2 inhibitor, further enhanced the improvement effects of ZKPC on bronchial asthmatic mice.

Various traditional Chinese medicines related to pingchuan have been demonstrated to improve asthma. Liu et al (39) indicated that pingchuan formula alleviated asthma in mice by restoring the balance of the T-helper cell17/regulatory $\mathrm{T}$ cell 

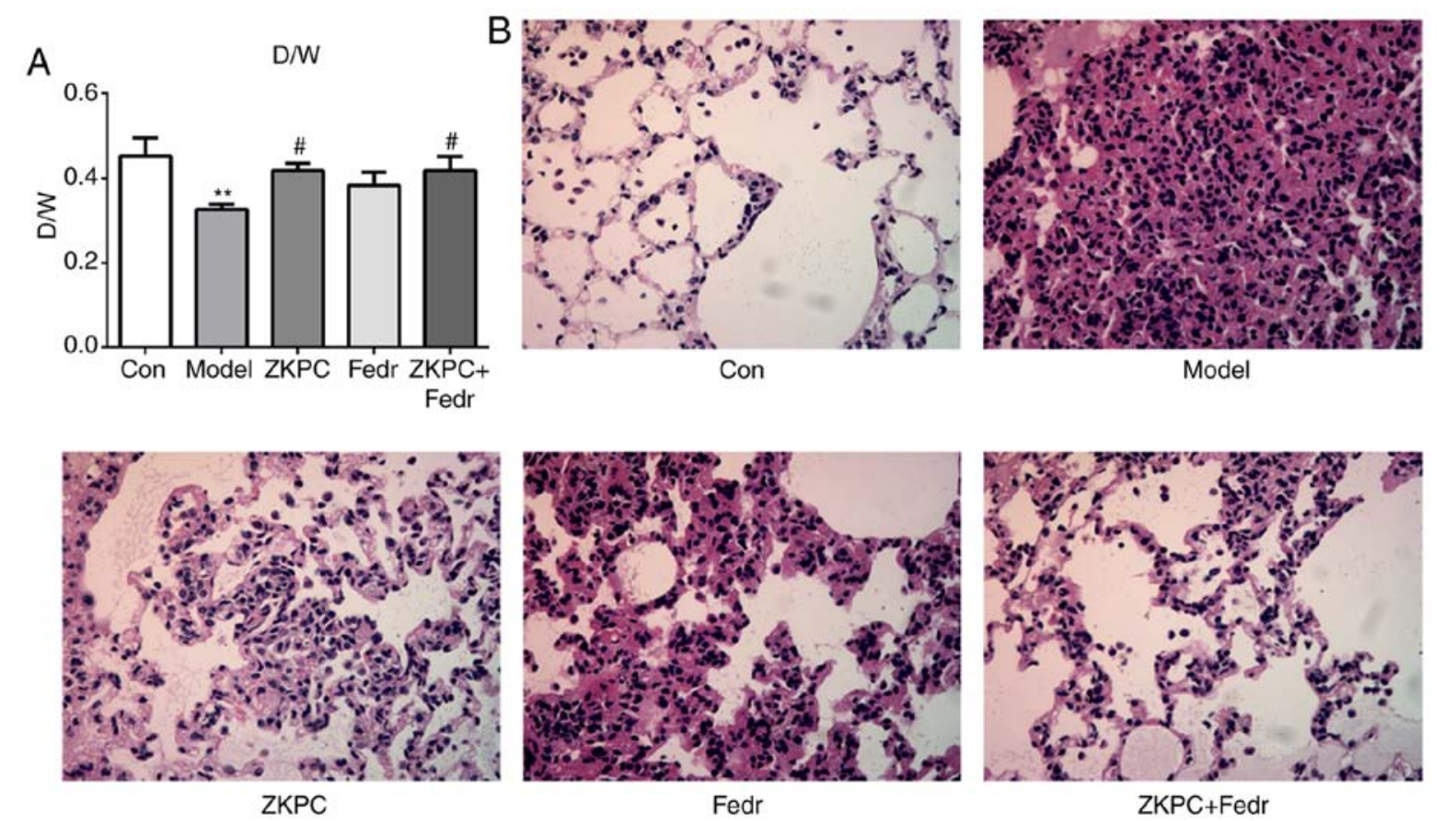

Figure 4. Fedratinib enhances the improvement effects of ZKPC on bronchial asthma-induced lung injury. (A) The D/W ratio of lung tissues was calculated after weighing. (B) Lung pathomorphology was observed by hematoxylin and eosin staining (magnification, $\mathrm{x} 400$ ). ${ }^{* *} \mathrm{P}<0.01$ vs. Con group. ${ }^{*} \mathrm{P}<0.05$ vs. Model group. $\mathrm{n}=10$. Fedr, fedratinib; Con, control; ZKPC, zhike pingchuan granules; L, low; H, high; D/W, dry/weight.
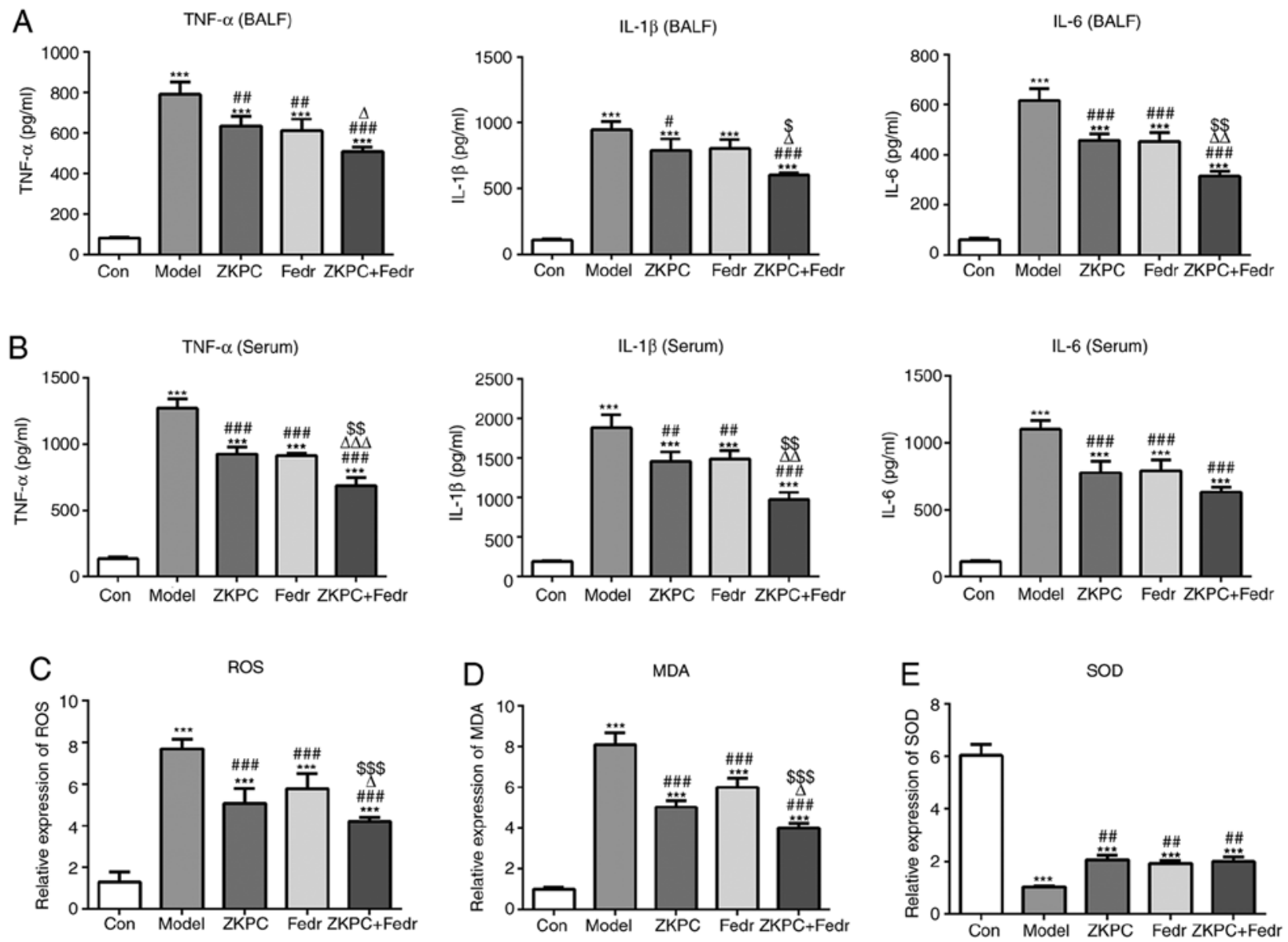

Figure 5. Fedratinib enhances the improvement effects of ZKPC on inflammation and oxidative stress in bronchial asthmatic mice. (A) The levels of IL-1 $\beta$, TNF- $\alpha$ and IL-6 in BALF and (B) serum were analyzed by ELISA. (C) ROS levels in lung tissues were detected using a ROS kit. (D) MDA levels in lung tissues was detected using an MDA kit. (E) SOD levels in lung tissues was detected using a SOD kit. $\mathrm{n}=10 .{ }^{* * *} \mathrm{P}<0.001$ vs. Con group. ${ }^{\# \mathrm{P}}<0.05,{ }^{\# \#} \mathrm{P}<0.01$ and ${ }^{\# \# \#} \mathrm{P}<0.001$ vs. Model group. ${ }^{\Delta} \mathrm{P}<0.05,{ }^{\Delta \Delta} \mathrm{P}<0.01$ and ${ }^{\Delta \Delta \Delta} \mathrm{P}<0.001$ vs. ZKPC group. ${ }^{\$} \mathrm{P}<0.05,{ }^{\$ \$} \mathrm{P}<0.01$ and ${ }^{\$ \$} \mathrm{P}<0.001$ vs. Fedr group. BALF, bronchoalveolar lavage fluid; ROS, reactive oxygen species; MDA, malondialdehyde; SOD, superoxide dismutase; ZKPC, zhike pingchuan granules; L, low; H, high; Con, control; Fedr, fedratinib. 

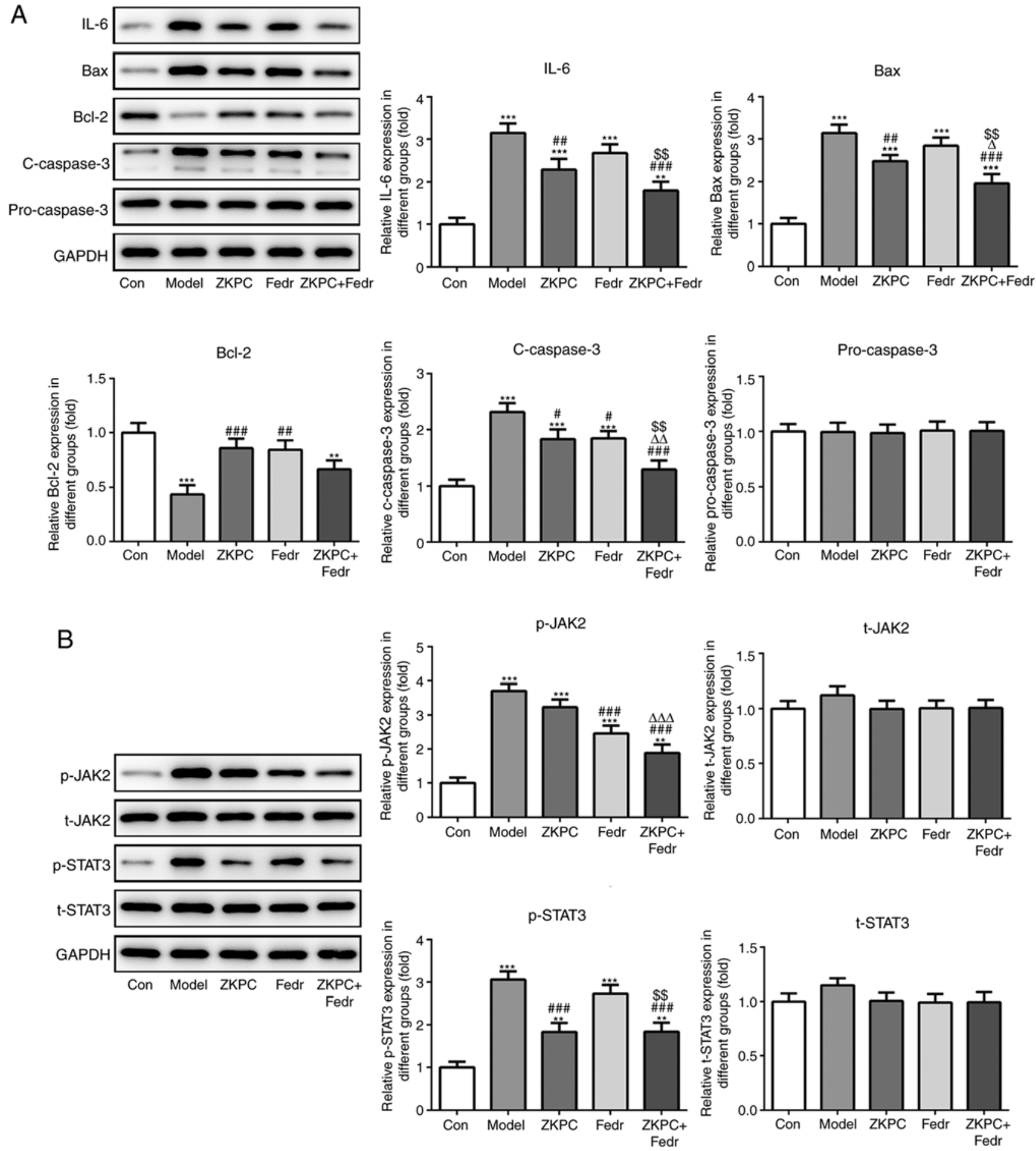

Figure 6. Fedratinib enhances the inhibitory effects of ZKPC on cell apoptosis and the IL-6/JAK2/STAT3 pathway in lung tissue. (A) The expression of IL-6, Bax, Bcl-2, c-caspase-3 and pro-caspase-3 and (B) levels of t-JAK2, p-JAK2, t-STAT3 and p-STAT3 in lung tissues were determined by western blot analysis. ${ }^{* *} \mathrm{P}<0.01$ and ${ }^{* * *} \mathrm{P}<0.001$ vs. Con group. ${ }^{\# \# \#} \mathrm{P}<0.001$ vs. Model group. ${ }^{\Delta \Delta \Delta} \mathrm{P}<0.001$ vs. ZKPC group. ${ }^{\$ \$} \mathrm{P}<0.01$ vs. Fedr group. $(\mathrm{n}=10){ }^{* *} \mathrm{P}<0.01$ and ${ }^{* * *} \mathrm{P}<0.001$ vs. Con group. ${ }^{\#} \mathrm{P}<0.05,{ }^{\# \#} \mathrm{P}<0.01$ and ${ }^{\# \# \#} \mathrm{P}<0.001$ vs. Model group. ${ }^{\Delta} \mathrm{P}<0.05,{ }^{\Delta \Lambda} \mathrm{P}<0.01$ and ${ }^{\Delta \Delta \Delta} \mathrm{P}<0.001$ vs. ZKPC group. ${ }^{\$ s} \mathrm{P}<0.01$ vs. Fedr group. c, cleaved; $\mathrm{t}$, total; p, phosphorylated; ZKPC, zhike pingchuan granules; L, low; H, high; Con, control.

ratio. Pan et al (40) found that Yanghe Pingchuan granules could ameliorate airway remodeling in a asthma rat model. Wang et al (41) indicated that Wenyang Yiqi Pingchuan improved lung pathomorphology and decreased the contents of nitric oxide and endothelin-1 in lung tissues of rats with bronchial asthma. These compounds contain ingredients which are also found in ZKPC. The present study found that ZKPC could alleviate lung pathomorphology, decreased the levels of oxidative stress and suppressed cell apoptosis in lung tissues of bronchial asthmatic mice.

In conclusion, ZKPC partially reversed the $\mathrm{D} / \mathrm{W}$ ratio and improved the lung pathomorphology of bronchial asthmatic mice. ZKPC inhibited inflammation, oxidative stress levels and cell apoptosis by suppressing the IL-6/JAK2/STAT3 pathway. In addition, the effects of ZKPC on bronchial asthma was further promoted by fedratinib treatment. 


\section{Acknowledgements}

Not applicable.

\section{Funding}

This study was supported by grants from the National Natural Science Foundation project: Experimental study on the regulation of TGF-1/VEGF expression in airway remodeling in asthmatic rats based on the new theory of 'hidden wind stagnation and lodge phlegm' pathogenesis (grant no. 81574020). Traditional Chinese Medicine Scientific Research Special Project of Henan Province (grant no. 20-21ZY2057); Henan Provincial Key Research and Development and Promotion Special Project (Science and Technology Targeted) (grant no. 212102311143)

\section{Availability of data and materials}

The datasets used and/or analyzed during the current study are available from the corresponding author on reasonable request.

\section{Authors' contributions}

YM designed the experiments and revised the manuscript. YR and YL performed the experiments and wrote the manuscript. YR, YL, SW, ZL, YY, XG, JH, SZ, HS, XT, QW, CC and YZ analyzed and interpreted the data. YR, YL, SZ and HS confirmed the authenticity of all the raw data. All authors read and approved the final manuscript.

\section{Ethics approval and consent to participate}

All animal experiments were approved by the Animal Experimental Ethics Committee of Henan University of Traditional Chinese Medicine (approval no. 20190412WZ).

\section{Patient consent for publication}

Not applicable.

\section{Competing interests}

The authors declare they have no competing interests.

\section{References}

1. Wu $\mathrm{J}$ and Hong $\mathrm{J}$ : Highlights of bronchial asthma in children guideline (2016 edition) for its diagnosis and treatment. World Clinic Drugs 39: 512-517, 2018 (In Chinese).

2. Masoli M, Fabian D, Holt S and Beasley R; Global Initiative for Asthma (GINA) Program: The global burden of asthma: Executive summary of the GINA Dissemination Committee report. Allergy 59: 469-478, 2004.

3. Alvarez-Alvarez I, Niu H, Guillen-Grima F and Aguinaga-Ontoso I: Meta-analysis of prevalence of wheezing and recurrent wheezing in infants. Allergol Immunopathol (Madr) 46: 210-217, 2018.

4. Liu SJ, Wang TT, Cao SY, Tan YQ and Chen LZ: A Meta analysis of risk factors for asthma in Chinese children. Zhongguo Dang Dai Er Ke Za Zhi 20: 218-223, 2018 (In Chinese).

5. Østergaard MS, Nantanda R, Tumwine JK and Aabenhus R: Childhood asthma in low income countries: An invisible killer? Prim Care Respir J 21: 214-219, 2012.
6. Lin JT, Wang WQ, Zhou X, Yin KS, Liu CT, Wang CZ, Huang M, Chen P, Yuan YD, Cai SX, et al: Trends of asthma control, disease management and perception in China. Zhonghua Jie $\mathrm{He}$ He Hu Xi Za Zhi 41: 191-195, 2018 (In Chinese).

7. Hsieh KH: Evaluation of efficacy of traditional Chinese medicines in the treatment of childhood bronchial asthma: Clinical trial, immunological tests and animal study. Taiwan Asthma Study Group. Pediatr Allergy Immunol 7: 130-140, 1996.

8. Wang R and Lin J: Analysis of the mechanism of Zhichuanling oral liquid in treating bronchial asthma based on network pharmacology. Evid Based Complement Alternat Med 2020: $1875980,2020$.

9. Wang L, Zheng X, Hui Y, Wang B, Yang Y, Feng X, Zhang T, Ma L and Zhang X: Adjuvant treatment with Xiaoqinglong formula for bronchial asthma: Protocol of systematic review and meta-analysis. Medicine (Baltimore) 98: e17053, 2019.

10. Su YH, Yu XY, Geng LM, Su KG and Guo YY: Oral administration versus iontophoresis of Sang-Su decoction (a formula for dispersing lung to calm panting and resolve phlegm and for dredging collateral) in the treatment of acute bronchial asthma: A comparative study on their efficacy and impacts on the prognosis. Int J Clin Exp Med 11: 1305-1311, 2018.

11. Jin X, Wang D, Xie J, Zhang L and Liu Q: Effect of Ginkgo Lactone on cholinergic neurotransmitters, oxidative stress and inflammation in $\mathrm{AD}$ model rats induced by $\mathrm{A} \beta_{1-40}$. J Chin Med Mater 11: 2693-2696, 2019 (In Chinese).

12. Thorpe LB, Goldie M and Dolan S: Central and local administration of Gingko biloba extract EGb $761^{\circledR}$ inhibits thermal hyperalgesia and inflammation in the rat carrageenan model. Anesth Analg 112: 1226-1231, 2011.

13. Li XH, Tu XY, Zhang DX, Xu JF, Wang WY, Zhang Y and $\mathrm{Du}$ YM: Effects of wuwei dilong decoction on inflammatory cells and cytokines in asthma model guinea pigs. J Tradit Chin Med 29: 220-223, 2009.

14. Wu WB, Zhu C and Luo C: Research on the protective effect of alkaloids Pinelliaf Rhizoma on inflammatory injury of lung epithelial cells. J Inner Mong Agric Univ 39: 1-8, 2018.

15. Su XD, Jang HJ, Li HX, Kim YH and Yang SY: Identification of potential inflammatory inhibitors from Aster tataricus. Bioorg Chem 92: 1-9, 2019.

16. Doganci A, Sauer K, Karwot R and Finotto S: Pathological role of IL-6 in the experimental allergic bronchial asthma in mice. Clin Rev Allergy Immunol 28: 257-270, 2005.

17. Martinez-Nunez RT, Bondanese VP, Louafi F, Francisco-Garcia AS, Rupani H, Bedke N, Holgate S, Howarth PH, Davies DE and Sanchez-Elsner T: A microRNA network dysregulated in asthma controls IL-6 production in bronchial epithelial cells. PLoS One 9: e111659, 2014.

18. Liu Z, Gan L, Zhou Z, Jin W and Sun C: SOCS3 promotes inflammation and apoptosis via inhibiting JAK2/STAT3 signaling pathway in 3T3-L1 adipocyte. Immunobiology 220: 947-953, 2015.

19. Imada $\mathrm{K}$ and Leonard WJ: The Jak-STAT pathway. Mol Immunol 37: 1-11, 2000.

20. Huang N, Liu K, Liu J, Gao X, Zeng Z, Zhang Y and Chen J: Interleukin-37 alleviates airway inflammation and remodeling in asthma via inhibiting the activation of NF- $\kappa$ B and STAT3 signalings. Int Immunopharmacol 55: 198-204, 2018.

21. Simeone-Penney MC, Severgnini M, Tu P, Homer RJ, Mariani TJ, Cohn L and Simon AR: Airway epithelial STAT3 is required for allergic inflammation in a murine model of asthma. J Immunol 178: 6191-6199, 2007.

22. Simon AR, Takahashi S, Severgnini M, Fanburg BL and Cochran BH: Role of the JAK-STAT pathway in PDGF-stimulated proliferation of human airway smooth muscle cells. Am J Physiol Lung Cell Mol Physiol 282: L1296-L1304, 2002.

23. Yan YR, Luo Y, Zhong M and Shao L: miR-216a inhibits proliferation and promotes apoptosis of human airway smooth muscle cells by targeting JAK2. J Asthma 56: 938-946, 2019.

24. Shi J, Li J, Yang S, Hu X, Chen J, Feng J, Shi T, He Y, Mei Z, $\mathrm{He} \mathrm{W}$, et al: LncRNA SNHG3 is activated by E2F1 and promotes proliferation and migration of non-small-cell lung cancer cells through activating TGF- $\beta$ pathway and IL-6/JAK2/STAT3 pathway. J Cell Physiol 235: 2891-2900, 2020.

25. Tang Y: Lipid cholesterol mediates the mechanism of arterial thrombosis by regulating the proliferation and activation of peripheral blood cells (unpublished $\mathrm{PhD}$ thesis). Jilin University, 2020.

26. Papi A, Brightling C, Pedersen SE and Reddel HK: Asthma. Lancet 391: 783-800, 2018. 
27. Brannan JD: Bronchial hyperresponsiveness in the assessment of asthma control: Airway hyperresponsiveness in asthma: its measurement and clinical significance. Chest 1138 (Suppl 2): 11S-17S, 2010

28. Sarnes E, Crofford L, Watson M, Dennis G, Kan H and Bass D: Incidence and US costs of corticosteroid-associated adverse events: A systematic literature review. Clin Ther 33: 1413-1432, 2011.

29. Papanicolaou DA, Wilder RL, Manolagas SC and Chrousos GP: The pathophysiologic roles of interleukin-6 in human disease. Ann Intern Med 128: 127-137, 1998.

30. Dimitrova D, Youroukova V, Ivanova-Todorova E, Tumangelova-Yuzeir K and Velikova T: Serum levels of IL-5, IL-6, IL-8, IL-13 and IL-17A in pre-defined groups of adult patients with moderate and severe bronchial asthma. Respir Med 154: 144-154, 2019.

31. Kado S, Nagase T and Nagata N: Circulating levels of interleukin-6, its soluble receptor and interleukin-6/interleukin-6 receptor complexes in patients with type 2 diabetes mellitus. Acta Diabetol 36: 67-72, 1999.

32. Kuhn C III, Homer RJ, Zhu Z, Ward N, Flavell RA, Geba GP and Elias JA: Airway hyperresponsiveness and airway obstruction in transgenic mice. Am J Respir Cell Mol Biol 22: 289-295, 2000.

33. Gavino AC, Nahmod K, Bharadwaj U, Makedonas G and Tweardy DJ: STAT3 inhibition prevents lung inflammation, remodeling, and accumulation of Th2 and Th17 cells in a murine asthma model. Allergy 71: 1684-1692, 2016.

34. Lv J, Sun B, Mai Z, Jiang M and Du J: STAT3 potentiates the ability of airway smooth muscle cells to promote angiogenesis by regulating VEGF signalling. Exp Physiol 102: 598-606, 2017.

35. Dickason RR, English JD and Huston DP: Engineering of a functional interleukin-5 monomer: A paradigm for redesigning helical bundle cytokines with therapeutic potential in allergy and asthma. J Mol Med (Berl) 74: 535-546, 1996.
36. Gong JH, Shin D, Han SY, Kim JL and Kang YH: Kaempferol suppresses eosionphil infiltration and airway inflammation in airway epithelial cells and in mice with allergic asthma. J Nutr 142: 47-56, 2012.

37. Nakano T, Inoue $H$, Fukuyama $S$, Matsumoto K, Matsumura M, Tsuda M, Matsumoto T, Aizawa $\mathrm{H}$ and Nakanishi Y: Niflumic acid suppresses interleukin-13-induced asthma phenotypes. Am J Respir Crit Care Med 173: 1216-1221, 2006.

38. Pitman H, Innes BA, Robson SC, Bulmer JN and Lash GE: Altered expression of interleukin-6, interleukin- 8 and their receptors in decidua of women with sporadic miscarriage. Hum Reprod 28: 2075-2086, 2013.

39. Liu F, Yu J, Bai L, Xue Z and Zhang X: Pingchuan formula improves asthma via restoration of the Th17/Treg balance in a mouse model. BMC Complement Altern Med 15: 234, 2015.

40. Pan LY, Han YQ, Wang YZ, Chen QQ, Wu Y and Sun Y: Mechanism of Yanghe Pingchuan granules treatment for airway remodeling in asthma. Drug Des Devel Ther 12: 1941-1951, 2018.

41. Wang $\mathrm{XH}$, Yang MX and Yu WT: Effect of Wenyang Yiqi Pingchuan recipe on pathomorphology of lung and its regulation on lung tissue contents of nitric oxide and endothelin-1 in rats with bronchial asthma. Zhongguo Zhong Xi Yi Jie He Za Zhi 29: 435-438, 2009 (In Chinese).

This work is licensed under a Creative Commons

Attribution-NonCommercial-NoDerivatives 4.0 International (CC BY-NC-ND 4.0) License. 